Archives de sciences sociales des religions

137 | janvier - mars 2007

Varia

\title{
Max Weber et les charismes spécifiques
}

\section{Régis Dericquebourg}

\section{OpenEdition}

Journals

Édition électronique

URL : http://journals.openedition.org/assr/4146

DOI : 10.4000/assr.4146

ISSN : $1777-5825$

Éditeur

Éditions de l'EHESS

\section{Édition imprimée}

Date de publication : 1 avril 2007

Pagination : 21-41

ISBN : 978-2-7132-2142-2

ISSN : 0335-5985

Référence électronique

Régis Dericquebourg, "Max Weber et les charismes spécifiques 》, Archives de sciences sociales des religions [En ligne], 137 | janvier - mars 2007, mis en ligne le 05 juin 2010, consulté le 20 avril 2019. URL : http://journals.openedition.org/assr/4146 ; DOI : 10.4000/assr.4146 


\section{Régis Dericquebourg}

\section{Max Weber et les charismes spécifiques}

On a beaucoup commenté les deux types de charisme proposés par Max Weber : le charisme prophétique et le charisme de fonction. Jean-Martin Ouedraogo a livré un récapitulatif raisonné et exhaustif ${ }^{1}$ de leurs attributs en désignant les commentateurs qui ont mis en relief chacun de ces derniers. On peut les rappeler. Le premier, charisme personnel ou charisme plénier, se définit par rapport à deux critères fondamentaux indissociables : "la qualité extraordinaire (à l'origine déterminée de façon magique tant chez les prophètes et les sages, thérapeutes et juristes, que chez les chefs de peuples chasseurs et les héros guerriers) d'un personnage pour ainsi dire doué de forces ou de caractères surnaturels ou surhumains en dehors de la vie quotidienne, inaccessibles au commun des mortels ${ }^{2}$ et sa reconnaissance par des suiveurs. Le porteur d'un charisme personnel s'écarte de l'ordre du quotidien et favorise la création de liens sociaux. En principe, sa survie dépend d'une économie aléatoire (offrandes, mécénat...), et il doit prouver (magiquement, héroïquement) ses dons extraordinaires par des succès (apport de bénéfices matériels ou immatériels). Le charisme a un caractère révolutionnaire par rapport aux époques et aux institutions. Il est mal reçu par les administrations et les autorités en place à cause de son caractère protestataire. Son porteur dicte des exigences nouvelles. Du point de vue sociologique, il désigne un mode de domination qui est en même temps un mode d'obéissance ${ }^{3}$ ainsi qu'un moteur du changement venant en temps de crise ${ }^{4}$. Par principe, les sociologues et les politologues mettent en relief les conditions sociales du caractère innovant $\mathrm{du}$ discours prophétique, le processus social de son acceptation, les causes de la

1. Voir Jean-Martin Ouedraogo, «La réception de la sociologie du charisme de M. Weber », in Archives de Sciences Sociales des religions, 83, 1993, pp. 141-157.

2. Max Weber, Économie et société, Paris, Plon (trad. sous la dir. de J. Chavez \& G. de Dampierre), 1971, p. 249. Voir Jean SÉGUY, «Charisme, sacerdoce, fondation : autour de Louis Grignion de Montfort », Social Compass, XXIX/I, 1982, pp. 5-24, cf. pp. 10-11. Voir aussi Jean SÉGUY, "Le clergé dans une perspective sociologique ou que faisons-nous de nos classiques ? in Prêtres, pasteurs et rabbins dans la société contemporaine, Paris, Cerf, 1982.

3. Raymond Aron, préface à Le Savant et le politique, Paris, Plon, 1959, p. 37.

4. Pierre Bourdieu, "Genèse et structure du champ religieux », Revue Française de Sociologie, XII, 1971, pp. 295-334, en particulier p. 333. Voir également p. 317 à propos du lien entre pouvoir symbolique et pouvoir prophétique. 
formation d'un mouvement de suiveurs et les transformations sociales qu'il produit ${ }^{5}$. Le second type, le charisme de fonction, est impersonnel. Il correspond à " une qualité exceptionnelle reconnue par une institution qui la porte à plénitude par confirmation rituelle; celle-ci fonde la légitimité du pouvoir auquel elle prétend faire accéder ceux qui l'ont reçue; elle les oblige à reproduire les obligations articulées par l'institution, propriétaire du charisme de fonction; ce dernier tire sa légitimité, en dernière analyse, de l'appropriation par une institution, d'un charisme personnel fondateur $»^{6}$.

Toutefois, certains charismes évoqués par Weber échappent aux deux types précédents. Par exemple, en traitant de la foi, Weber souligne l'existence d'un charisme qu'il dit spécifique ${ }^{7}$ et qu'il nomme le charisme de la foi ${ }^{8}$. Dans ce cas, il n'évoque ni le charisme du prophète, ni le charisme institutionnel détenu par un clerc mais un charisme qui semble spécifier une qualité ou une capacité particulière reconnue à son porteur. Dans le même ordre d'idée, on trouve aussi sous sa plume le charisme de la bonté, le charisme de la raison, le charisme du savoir-faire, le charisme de l'esprit et du goût, le charisme de la chasteté, le charisme de la vertu, le charisme de la certitude du salut, le charisme de l'illumination qui ne sont pas qualifiés de spécifiques par l'auteur mais que nous pouvons considérer comme tels car ils correspondent au même souci de spécification que le charisme de la foi.

Dans cet article, nous évoquerons cette catégorie de charisme déjà remarquée, bien qu'il ne l'ait pas développée, par Joachim Wach à propos du fondateur de religion et du réformateur ${ }^{9}$. Nous tenterons de découvrir ce que Weber entend par charisme spécifique, notion qu'il ne définit pas et dont il ne fait pas un type idéal. Nous voulons montrer que ces charismes d'un troisième genre ne légitiment pas forcément une domination et rendent la compréhension du charisme plus compliquée que ne l'ont imaginé certains auteurs. Nous pensons que les charismes spécifiques fondent une virtuosité dans un domaine de l'action quotidienne plus que dans l'extra-quotidienneté typique du charisme prophétique. Nous nous

5. Pierre Bourdieu, "Une interprétation de la théorie de la religion selon Max Weber ", Archives européennes de sociologie, XII-1, 1971, pp. 3-21.

6. Jean SÉGUY, "Le clergé dans une perspective sociologique ", in Prêtres, Pasteurs et Rabbins dans la société contemporaine, op. cit., p. 40.

7. Économie et société, op. cit., p. 576. Également dans Sociologie des religions, Paris, Gallimard (trad. J.-P. Grossein), 1996, p. 227, section III : «Voies de salut, leur influence sur la conduite de la vie », pp. 177-240.

8. Économie et société, op. cit., pp. 577 et 580.

9. Selon Joachim Wach, l'activité du fondateur de religion " peut s'accompagner des fait miraculeux tels que les guérisons, distributions de nourriture, métamorphose (...). Ces actes ont été regardés comme autant d'exemples mettant en valeur le charisme spécifique qui accrédite l'homme de Dieu sans risque d'erreur possible » (J. Wach, Sociologie de la religion, Paris, Payot, 1955, p. 302-303). À la page 304 l'auteur ajoute : "Les réformateurs n'ont pas tous le même charisme spécifique ». Il mentionne comme tel le don de vision ou d'extase, la qualité prééminente du don de l'esprit et du cœur, un talent exceptionnel d'organisation ou une ascèse vigoureuse comme charismes spécifiques. 
demanderons s'ils sont utiles à l'interprétation des activités sociales. Pour étayer nos considérations, nous nous attarderons sur le charisme de la bonté (Charisma der Güte) car Weber livre à son propos, directement ou indirectement, plus d'information que sur les autres charismes spécifiques et aussi parce qu'il offre de nombreuses illustrations historiques. Parmi celles-ci, nous avons retenu saint Vincent de Paul qui a consacré sa vie à la charité. Nous revisiterons également le charisme des thérapeutes des «Églises de guérison » qui peuvent fournir une base empirique justifiant notre idée.

\section{Les divers charismes spécifiques}

Si l'auteur de Économie et société ne définit pas précisément les charismes spécifiques, on s'aperçoit que ceux-ci trouvent un sens lorsqu'il sont mis en perspective. Chez Weber, le charisme de la raison, appliqué à Robespierre, est la forme ultime prise par le charisme au cours de son histoire riche en événements ${ }^{10}$ car elle repose sur la croyance "propre à l'époque des Lumières » selon laquelle la raison individuelle "doit donner naissance à un monde qui serait au moins relativement, le meilleur possible grâce à la providence divine, et cela parce que l'individu est celui qui connaît le mieux ses propres intérêts. " ${ }^{11}$. Ce charisme est la transfiguration ${ }^{12}$ d'un charisme religieux. Il devient le principe transcendant d'une régénération quasi messianique ${ }^{13}$ du monde (Est-ce par humour que Weber le soumet à la providence divine ?). L'idéalisation et l'illumination charismatique de la Raison ont trouvé leurs glorifications pendant la Révolution de 1789 puis dans le culte de la raison ${ }^{14}$, une raison qui n'est plus seulement un mode d'explication du monde et un guide pour l'action mais qui est magnifiée. Dans les lignes que Weber consacre à Robespierre, le charisme de la raison semble, pour l'occasion, le principe d'un charisme révolutionnaire qui légitime la domination des " gouvernants révolutionnaires ». En ce cas, « la direction administrative personnelle est recrutée charismatiquement parmi les plébéiens (...) doués, dans le cas de Robespierre, de certaines qualités "éthiques" à côté de la confiance personnelle ${ }^{15}$ des masses. La raison charismatique est plus que l'exercice individuel de la raison. Elle est une vertu que revendique Robespierre pour légitimer sa domination.

Avec le charisme du savoir-faire ${ }^{16}$, Weber évoque la création artistique qui évolue vers la recherche de formes selon une quête esthétique qui a sa logique

10. Max Weber, Sociologie des religions, op. cit., p. 327-328.

11. Idem, p. 327.

12. Jean-Martin OuedRAOGO, "Le charisme selon Max Weber : la question sociologique ", Archives européennes de sociologie, XXXVIII, 1997, p. 340.

13. Eugen Weber, Apocalypses et millénarismes, Paris, Fayard, 1999, pp. 128-129.

14. Cf. Matthiez, La théophilanthropie et le culte décadaire, Paris, 1903.

15. Max Weber, Économie et société, op. cit., p. 276.

16. Max Weber, Sociologie des religion, op. cit., p. 435. 
intrinsèque et son univers de valeurs esthétiques profanes, au détriment d'un art dont le sens est fixé par une tradition religieuse. Weber écrit : "Toute religiosité sublimée du salut-délivrance ne considère que le sens et non la forme des choses et des actes qui importent pour le salut. À ses yeux, la forme est ravalée au niveau de l'accidentel, de la créature, de ce qui détourne du sens. Du côté de l'art, le rapport ingénu aux choses peut, certes, rester intact ou constamment se reconstituer, aussi longtemps et aussi souvent que l'intérêt conscient du récepteur de l'œuvre d'art s'attache naïvement au contenu de ce qui a pris forme, et non à la forme perçue pour elle-même; et aussi longtemps que la réalisation du créateur est perçue soit comme un charisme (magique, à l'origine) du savoirfaire, soit comme un libre jeu ${ }^{17}$. Le charisme du savoir-faire s'applique à un art inspiré par une tradition religieuse qui s'attache à la «sublimation de l'éthique religieuse et de la recherche du salut ${ }^{18}$. Sinon, il se situe dans le domaine profane de l'esthétique où des amateurs exigent de l'artiste une technicité et des thèmes originaux. Georg Simmel nous aide à comprendre ce type de charisme. Pour lui, l'art et la religion sont dissociés, voire en tension, puisque l'art peut faire concurrence à la religion en distrayant l'homme de la quête de Dieu ; toutefois, il existe des dimensions religieuses de l'expression artistique qui ne passent pas uniquement par des scènes religieuses (telles les "nativités ") mais qui, comme chez Rembrandt, passent par des représentations de la vie quotidienne d'où émane une piété ; pour Simmel l'âme y est "délocalisée » au sens où ce ne sont plus les personnages du tableau qui « sont religieux $"{ }^{19}$ mais la scène elle-même, empreinte de spiritualité. Des personnages « ordinaires "sont spiritualisés comme l'est la souffrance dans la peinture chrétienne, souffrance dégagée de la dépression et de la laideur au point de devenir une nouvelle valeur ${ }^{20}$. Simmel ne rejoint pas tout à fait Weber mais il explique ce qu'est un art transfiguré religieusement stimulant «la disposition religieuse » et, finalement, il illustre, avec l'exemple de Rembrandt, le charisme du savoir-faire qui n'est pas qualifié comme tel ${ }^{21}$ et qui est reconnu à l'artiste dont l'art est religieusement sublimé.

Le charisme de l'esprit et $d u$ goût est plus délicat à expliquer. Il renvoie à l'attachement aux biens matériels qui s'exprime par un hédonisme séculier et par la recherche du raffinement comme chez les dandys, chez les passionnés de la littérature et des arts et chez ceux pour qui la culture transcende la vie quotidienne. Weber situe cet élan psychologique dans une théodicée où s'exprime un attachement aux valeurs temporelles au détriment de l'attachement aux valeurs intemporelles ${ }^{22}$.

17. Idem, p. 435.

18. Idem, p. 435.

19. Danielle Hervieu-Léger et Jean-Paul Willaime, Sociologies et religion, Paris, PUF, 2001, pp. 138-141.

20. Idem, p. 140.

21. Georg Simmel, Rembrandt, Saulxsures, 1994 (1916).

22. Max Weber, Sociologie des religions, op. cit., p. 453. 
De l'esprit et du goût, Weber passe aux valeurs qui guident leurs activités et qui seraient la poursuite des vanités proposées par la culture profane dans le monde et qui, dans certaines théologies comportant une morale de la modération (en particulier dans le christianisme), détourneraient l'homme du salut et entreraient en concurrence, voire en conflit avec le détachement du temporel comme valeur. Dans une société fortement imprégnée par le rejet de l'hédonisme séculier, la tension entre la religion et la culture laïque risque d'être grande et le porteur d'un charisme de l'esprit et du goût serait celui qui excellerait à persévérer dans la jouissance des biens culturels profanes. Cette fois, nous constatons qu'une pulsion vers le monde devient un charisme à partir de sa sublimation par la culture et par une philosophie de l'hédonisme qui s'oppose à la théodicée du refus du monde.

Le charisme de la foi apparaît dans les paragraphes du chapitre : "Les voies du salut et leur influence sur la conduite de la vie " consacrés à la relation aux sauveurs en ces termes : "Mais plus originelle encore est la conception, déjà mentionnée que l'on trouve aussi dans le Nouveau Testament, de la foi en tant que charisme spécifique d'une confiance extraordinaire et purement personnelle dans la providence de Dieu, telle que doivent la posséder les pasteurs des âmes et les héros de la foi. " ${ }^{23}$. À propos de ces derniers, Weber affirme qu' " en vertu de cette confiance charismatique dans le soutien de Dieu qui dépasse les formes humaines ordinaires, l'homme de confiance de la communauté émotionnelle, en tant que virtuose de la foi, peut pratiquement agir et accomplir d'autres choses que le laïc ${ }^{24}$. La foi procurerait une «sorte de succédané des facultés magiques ". Le charisme de la foi n'est donc pas détenu par n'importe lequel des hommes religieux mais par ceux qui l'ont éveillé ( «la foi chrétienne en la Providence est un charisme qui doit être établi par l'exercice de la volonté du croyant » ${ }^{25}$ ) grâce à la mortification de l'orgueil intellectuel. En rappelant que le charisme de la foi a été reconnu par Jésus aux petits enfants et non aux savants ${ }^{26}$, l'auteur souligne la tension entre le christianisme et l'intellectualisme. Il est antirationnel et peut conduire à une confiance inconditionnelle en la Providence divine et à une virtuosité religieuse. Il spécifie une vertu native ou éveillée par une ascèse reconnue chez un individu. Son porteur l'a en commun avec le prophète bien qu'il n'en soit pas un, mais il peut devenir un héros c'est-à-dire l'autre grand type de personnage doté d'un don exceptionnel chez Weber.

On trouve le même abandon à une transcendance dans le charisme de la certitude du salut. Cette expression apparaît dans les considérations de Weber sur les voies de salut: "La prédestination garantit à celui qui bénéficie de la

23. Max Weber, Économie et société, op. cit., p. 576.

24. Idem, p. 576.

25. Idem, p. 580 .

26. Idem, p. 577. 
grâce le suprême degré de la certitude du salut dès qu'il est sûr d'appartenir à l'aristocratie restreinte du salut, de compter au petit nombre des élus. Quand l'individu possède ce charisme d'une importance sans égale, il doit en exister des symptômes - car l'incertitude absolue est insupportable à la longue. » ${ }^{27}$. Ces symptômes ne peuvent résider que dans l'accomplissement des commandements divins en tant qu'instrument de Dieu - « continuement et méthodiquement car on possède la grâce ou toujours ou jamais » ${ }^{28}$. Il s'agit d'un charisme religieux actif qui découle d'une relation intime et authentique à Dieu " fondé sur le rapport secret de la grâce ${ }^{29}$ qualité centrale et constante de la personnalité qui donne la certitude du salut et de la persévérance dans la grâce. Plus loin, Weber lie les conduites qui en découlent à la virtuosité dans l'acquisition chez les Puritains ${ }^{30}$. Le charisme de la certitude et du salut se rapporte à l'homme qui se fait instrument permanent de Dieu dans le monde grâce à une ascèse intramondaine, en Occident, dans le cadre d'un métier exercé comme une "vocation » ${ }^{31}$ profane et qui devient un « virtuose de la vertu professionnelle exercée dans le monde » 32 . L'homme de la certitude de salut éveille ce charisme en agissant avec persévérance selon la volonté de Dieu qu'il trouve dans l'intériorisation des commandements divins et le soumet à son Église. Il n'impose aucune obligation nouvelle, n'agit pas en fonction d'un statut ecclésial mais il s'emploie à faire son salut d'une manière exemplaire.

Weber affirme également que la grâce de la certitude du salut dévalorise radicalement la grâce magique et institutionnelle. Ce charisme n'est donc ni celui du prophète, ni celui du magicien, ni celui du clerc. L'expression « charisme de la certitude du salut » spécifie la conduite éthique de l'homme assuré de posséder la grâce nécessaire au salut.

Le charisme de la chasteté est mentionné dans le chapitre sur l'éthique et le monde : "Pour Saint Paul, la dignité de la continence absolue était un charisme purement personnel des virtuoses religieux ${ }^{33}$. Ici, la chasteté est légitimée par la volonté de surmonter les instincts sexuels, ce que ne peut faire "l'homme moyen ». Elle a pour conséquence le renoncement à la fondation d'une famille et l'exclusivité du lien avec Dieu. On peut aussi la relier à une ascèse qui passe par la sublimation des désirs ou, comme dans le yoga, par le détournement d'une énergie et le rejet de plaisirs illusoires. En aucun cas, la chasteté involontaire produite par la solitude, la maladie physique ou mentale n'est un charisme. Il faut qu'elle soit volontaire et incluse dans une ascèse pratiquée en vue du salut.

\author{
27. Idem, p. 581. \\ 28. Idem, p. 581. \\ 29. Idem, p. 581. \\ 30. Idem, p. 582. \\ 31. Max Weber, Sociologie des religions, op. cit., p. 363. \\ 32. Idem, p. 192. \\ 33. Max Weber, Économie et société, op. cit., p. 608.
}


Le charisme de la vertu ${ }^{34}$ est appliqué au charisme "non modifié dans le sens charismatique héréditaire ${ }^{35}$ du monarque de la Chine. Weber traite d'une qualification charismatique si absolue que des sanctions frappaient le monarque quand des malheurs s'abattaient sur son pays. Il ne suffisait pas que celui qui préside aux destinées du Royaume ait des « dispositions à faire le bien » (vertueux), il devait avoir un pouvoir extraordinaire en qualité de "fils du ciel » légitime. La vertu est charisme dans la mesure où son porteur a reçu une grâce de l'Esprit du Ciel. Il lui était demandé d'avoir des dons magiques de type apotropaïque : écarter les désastres de la guerre, apporter la pluie (contrer la sécheresse), l'éloigner (contrer les inondations) ou influencer le cours des astres. Bien que lié à une fonction héritée, car il n'exige aucune confirmation, ce charisme spécifie une grâce qui, confirmée, légitimait la place du monarque dans une fonction héritée.

L'expression charisme de l'illumination est employée par Weber lorsqu'il évoque la tension entre le savoir religieux et la connaissance intellectuelle ${ }^{36}$. L'auteur en traite dans Hindouisme et Bouddhisme ${ }^{37}$. La religion prétend que la connaissance qu'elle procure est d'un autre ordre que la "suffisance agressive de l'intellect " ${ }^{38}$. Au savoir intellectuel ultime concernant l'état ou le domaine normatif, la religion oppose une prise de position ultime à l'égard du monde en vertu d'une saisie immédiate de son sens appelé charisme de l'illumination. Ce dernier serait réservé uniquement aux personnes qui se libéreraient « des succédanés trompeurs que les impressions confuses du monde sensible et les abstractions de l'entendement " ${ }^{39}$ proposent en guise de connaissance alors qu'elles sont "vides et indifférentes » au regard du salut. On atteindrait l'illumination en ménageant en soi une place pour accueillir «l'essentiel » : le sens du monde et de sa propre existence. L'illumination n'est pas un libre présent de la grâce divine. Elle est salaire d'une ascèse contemplative méthodique. Elle produit un état de félicité considéré ici-bas comme un état de grâce (donc un charisme qui n'a pas à être confirmé par une action dans le monde). Celui qui l'atteint a la certitude d'être définitivement détaché de la "soif de vivre » et rejoint en ce sens la sainteté ${ }^{40}$. Le charisme de l'illumination qu'on trouve dans les biographies de maîtres spirituels hindous serait donc un "insight " obtenu grâce à des exercices spirituels ou corporels (de type yoga). Il est la réalisation aboutie de l'application d'une technique qui transfigure une réalité par le sens. Il produit une nouvelle saisie du sens du monde. Ainsi naît la perception du monde matériel comme une illusion.

34. Idem, p. 250.

35. Idem, p. 250.

36. Dans les "Considérations intermédiaires" in Max WeBER, Sociologie des religions, op. cit., pp. 450-451.

37. Max WeBER, Hindouisme et Bouddhisme, Paris, Flammarion, (trad. I. Kalinowski et R. Lardinois), 2003, pp. 371-372.

38. Max Weber, Sociologie des religions, op. cit., p. 450.

39. Idem, p. 450-451.

40. Max Weber, Hindouisme et Bouddhisme, op. cit., p. 372. 
C'est une expérience mystique difficilement communicable. Nous constatons que le charisme de l'illumination peut être à l'origine d'un prophétisme : c'est le cas du Bouddha qui, après sa "nuit de l'éveil » décide d'annoncer une nouvelle voie de salut et de livrer les techniques permettant de l'atteindre. Mais en dehors de cas extrêmes, la courte description que Weber fait de ce charisme permet de le considérer d'un point de vue plus ordinaire. En évoquant le charisme de l'illumination, Weber semble ne s'intéresser qu'aux religions asiatiques en omettant l'illumination mystique chrétienne ${ }^{41}$ et musulmane ${ }^{42}$.

Le charisme de l'état de grâce est évoqué ${ }^{43}$ à propos de l'appartenance à une communauté et de l'occupation d'une charge à l'intérieur de sectes opposées à la domination des théologiens. Dans ces mouvements, le ministre n'exerce pas une fonction de "mercenaire». Sa charge est généralement une occupation secondaire bien qu'elle puisse être détenue à temps plein par des missionnaires considérés à l'égal des autres laïcs. La domination des laïcs s'exprime par la revendication de la libre prédication et, comme chez les baptistes, par un contrôle moral qui fait que les laïcs deviennent en quelque sorte "cléricalisés ». Weber l'illustre en prenant l'exemple des Quakers où chacun peut parler à l'assemblée religieuse, à condition que l' «Esprit » soit descendu en lui. L'existence d'un clergé professionnel est donc exclue ${ }^{44}$. Ceux qui acceptent une charge, ou qui sont candidats à une charge, dans la communauté ne possèdent pas un charisme de fonction impersonnel et s'ils ont reçu une formation théologique, celle-ci n'est qu'une « condition technique préalable ${ }^{45}$. Ce qui l'emporte dans la désignation des laïcs à une charge est un charisme de l'état de grâce reconnu par les fidèles. Il peut être constaté par des enquêtes aboutissant parfois à la délivrance de " certificats de qualification religieuse ». Ce charisme "seul » importe et passe avant la fonction et l'instruction et peut même leur être opposé ${ }^{46}$. Le recours au seul état de grâce pour qualifier les prédicateurs ne fut pas toujours durable. Dans une note ${ }^{47}$, Weber rappelle que le méthodisme a, d'abord, aboli toute différence entre les prédicateurs ayant reçu l'ordination dans l'Église anglicane et ceux qui ne l'avaient pas reçu. Mais, peu à peu, les prédicateurs se sont transformés en un nouveau clergé et en 1836, une ordination formelle fut instituée à leur intention. Ceux dont le prêche n'était qu'une activité secondaire ne purent

41. Voir, par exemple, PASCAL, "Nuit mystique du 23 novembre 1654 » in Le mémorial. Euvres complètes de Pascal, section IV, Paris, Seuil, 1963, p. 618 ; Emmanuel Renault, Sainte Thérèse d'Avila et l'expérience mystique, Paris, Seuil, 1970, pp. 22, 14, 47, 92-93 ; Alain de LiberA, Maître Eckhart et la mystique rhénane, Paris, Cerf, 1999, p. 39.

42. Marijan Molé, Les mystiques musulmans, Paris, PUF, 1965, pp. 55-57, 61, 65, 91, 95,99 .

43. Max Weber, Éthique protestante et esprit du capitalisme, Paris, Plon, (trad. J. Chavy), 1964, pp. 283-286.

44. Idem, p. 284.

45. Idem, p. 286.

46. Idem, p. 284.

47. Idem, p. 285, note 50 . 
administrer les sacrements. Le cas est spécifique car la Mission chrétienne (future Armée du salut) fondée par William Booth disposait de prédicateurs, masculins et féminins, à temps plein ou partiel traités à égalité, situés les uns et les autres en dehors de la pratique sacramentelle et auxquels elle attribuait des charges de gestionnaires. On le voit, le charisme de l'état de grâce est un charisme reconnu à des laïcs transfigurés par une conversion à l'intérieur d'un mouvement religieux. Dans les sectes qui rejettent le recours à un clergé professionnel, il serait le succédané du charisme de fonction mais ce dernier est institutionnel alors que le charisme de l'état de grâce est reconnu à certaines personnes par les fidèles.

On trouve la mention du charisme de la bonté dans le paragraphe 10 de la Sociologie des religions intitulé "Les voies du salut et leur influence sur la conduite de la vie ${ }^{48}$ et plus précisément dans l'examen des prestations sociales comme œuvres personnelles de salut. On lit: «Il en va de même de toutes les prestations sociales quel que soit l'aspect qu'elles puissent revêtir ; si ce sont celles de "l'amour du prochain", la systématisation exige la possession du charisme de la "bonté" " 49 . Plus haut, Weber précise que ces prestations sociales peuvent être des œuvres attachées à l'" amour du prochain ", qu'une "systématisation peut s'instituer (et...) que la fonction normale de la prophétie est de la trouver $"{ }^{50}$. Weber enchaîne en parlant d'une éthique des « bonnes œuvres » qui peut prendre deux caractères différents. Le premier, celui du compte courant où s'inscrivent les bonnes actions réalisées de temps à autre et qui sera estimé à la mort pour accorder le salut. Le second, qui nous intéresse ici, une systématisation éthique qui ne traite les prestations individuelles qu'en tant que "symptôme et expression d'une personnalité éthique correspondante " ${ }^{51}$ révélant la personne dans sa totalité. Dans les lignes suivantes, nous apprenons qu'il en résulte un habitus total de la personnalité considéré comme un don de Dieu ou comme le fruit d'un entraînement que nous pourrions appeler un noviciat du bien. Cet habitus personnel des «bonnes œuvres » prend la place de la sanctification formelle par les œuvres au moyen d'actions extérieures singulières ${ }^{52}$. La pratique des «bonnes œuvres» exercée de manière permanente serait un instrument au service du salut, en même temps qu'une ascèse puisqu'elle nécessite un effort sur soi-même.

La cause des prestations sociales « quelque soit l'aspect qu'elles peuvent revêtir ", se trouve non pas dans la simple bonté (dimension psychologique) mais dans la possession du charisme de la «bonté ». Celui-ci est lié à l'amour du prochain, à l'éthique des "bonnes œuvres ", au salut, à une grâce ou à une ascèse qui engage la personnalité toute entière. On constate aussi que Weber ne s'intéresse pas à la qualité du porteur du charisme mais à la dynamique religieuse qu'il lance.

\footnotetext{
48. Max Weber, Économie et société, op. cit., p. 548.

49. Idem, p. 548.

50. Idem, p. 547.

51. Idem, p. 548.

52. Idem, p. 548.
} 


\section{L'exemple du charisme spécifique de la bonté chez saint Vincent de Paul}

Saint Vincent de Paul (1581-1660) illustre un charisme de la bonté qui soulève des élans de charité et s'institutionnalise sous la forme d'ordres religieux (Filles de la charité, Lazaristes). Chez ce clerc, la charité pourrait être une mission liée au charisme de fonction tel que généralement entendu. En effet, il ne manque pas de prêtres qui réunissent autour d'eux un groupe de fidèles se consacrant dans la mesure de leurs possibilités aux pauvres, aux personnes souffrantes ou aux esseulés. Le cas n'est pas exceptionnel dans l'histoire. Toutefois, si l'on en croit ses biographes, on trouve chez saint Vincent de Paul quelque chose de plus qu'un sens de la solidarité chrétienne et qui pourrait aider à comprendre le charisme de la bonté. Pour Jean Calvet, " la charité de Vincent est assurément vertu surnaturelle commandée par la loi évangélique... » ${ }^{53}$ qui se manifeste par les bonnes œuvres. L'auteur ajoute: "Partout où il missionne, il établit la charité, une confrérie bien organisée, un instrument à la fois bien réglé et souple pour secourir les pauvres et les malades non pas à coup de générosités spectaculaires, mais d'une manière permanente et pratique ". C'est une sorte de "ministère de l'assistance qui commence à se constituer, qui pose ici et là ses services et les étendra peu à peu à tout le royaume ${ }^{54}$. Autrement dit, saint Vincent de Paul préconise un système social des bonnes œuvres plutôt que des actes de charité isolés; sa charité est l'expression d'un charisme qui la rend "surnaturelle » (opera spiritualia) mais, en plus il éveille chez ses suiveurs un habitus permanent de charité en leur rappelant qu'il faut "se donner à Dieu pour servir les pauvres... » et que "Jésus représentant les pauvres, son Église doit s'organiser pour eux $"{ }^{55}$. Pour lui, les bonnes œuvres s'inscrivent dans la voie du salut. Il écrit : "Nous ne pouvons pas mieux assurer notre bonheur éternel qu'en vivant et mourant au service des pauvres entre les bras de la Providence et d'un actuel renoncement de nous-mêmes pour suivre Jésus-Christ » ${ }^{56}$. Parce qu'il renouvelle un commandement religieux et qu'il produit des effets collectifs de charité, on peut considérer qu'il illustre le charisme de la bonté déjà évoqué.

La mission de charité de saint Vincent de Paul semble osciller entre le charisme de fonction qu'il détenait normalement en tant que prêtre et un charisme personnel privé qui est précisément celui de la bonté. Comme Louis Grignion de Montfort, saint Vincent de Paul ne revendique jamais un mandat divin pour asseoir sa légitimité. Jeune prêtre, il ne rêvait que de se faire une situation honorable et rentable dans une "bonne " cure. Bérulle, son directeur de conscience en fit le confesseur de plusieurs hauts personnages du Royaume, tâche qu'il accomplit

53. Jean Calvet, Saint Vincent de Paul, Paris, Albin Michel, 1948, p. 55.

54. Idem, p. 89.

55. André Dodin, Saint Vincent de Paul, Paris, Seuil, 1960, pp. 65-66, 69.

56. In André Dodin, "Lettre à Jean Barreau, prêtre de la mission 4 décembre 1648-III ", p. 392 . 
pour l'Église. Soumis à celle-ci, il fit néanmoins preuve d'une certaine originalité dans l'interprétation de son ministère. Pour lui, relancer la pratique dans une paroisse, confesser, distribuer la grâce à travers le système sacramentel ne suffisait pas à la vie chrétienne telle qu'il l'entendait. Ses rencontres avec la misère, avec la faim, avec les enfants abandonnés, avec les galériens, le conduisirent à penser que «la foi qui sauve c'est celle qui agit et s'épanouit en charité, en amour effectif des hommes et de Dieu » et "par là il en vint à la charité » ${ }^{57}$. Il ne croit pas en la vertu de charité individuelle et occasionnelle mais pense que la charité doit être communautaire et organisée pour être efficace et durable. Pour cette raison, il veut l'asseoir sur des confréries. Il fonde la congrégation des Filles de la charité et celle des Lazaristes qu'il subordonne à l'Église. Pour lui, la cure d'âme (confession, sacrements) doit accompagner les soins du corps. Les fondations de saint Vincent de Paul ne sont pas des aventures hors de l'Église ; au contraire, elles renouvellent une des missions mal remplies à son époque. De ce fait, elles accroissent les services de l'Église au peuple et en élargissent l'influence. La charité est traditionnelle dans l'Église où elle est la première des vertus ${ }^{58}$ mais il fallait qu'elle soit soutenue par une force hors du commun pour fonder des congrégations charitables en surmontant les difficultés matérielles. Pour cela, il faut plus à Vincent de Paul qu'un charisme de fonction. N'étant ni celui-ci, ni charisme prophétique, son charisme de la bonté peut dès lors être considéré comme un charisme personnel privé tel que nous le définissons plus bas. La bonté est sublimée et devient une force peu commune qui met en œuvre une organisation destinée à améliorer la condition de l'homme, qui pousse son porteur à demander la justice sociale prenant le relais de la charité et qui fait de lui un pèlerin de la paix demandant aux grands personnages du Royaume l'arrêt des guerres civiles dévastatrices ${ }^{59}$.

Au nombre des attributs charismatiques, ses biographes lui reconnaissent une autorité personnelle. À Foleville (25 janvier 1617), il touche si fort les habitants en les exhortant à la vie chrétienne qu'il faut l'aide des jésuites d'Amiens pour confesser, prêcher et catéchiser. À Chatillon-les-Dombes, où il crée une confrérie de charité, il relance la pratique religieuse chez les habitants et ramène les ecclésiastiques "défaillants " à leurs fonctions car il voit en eux de possibles ennemis de l'Église ${ }^{60}$. Il a aussi une véritable autorité sur les personnes dont il est le confesseur et sur les membres de ses confréries. Il possède un don de la parole qui attire les premières Dames de la charité parmi les personnes fortunées comme

57. Michel Riquet, Saint Vincent de Paul ou le réalisme de la charité, Paris, Gabalda et Cie, 1960, p. 50.

58. Qui avait déjà engendré des hôpitaux, cf. René PiacentinI, Origine et évolution de l'hospitalisation. Les chanoinesses de la Miséricorde de Jésus, Paris, Grasset, 1957.

59. Robert Sabatier, Saint Vincent de Paul, Paris, La Table Ronde, 1959.

60. Michel Riquet, Saint Vincent de Paul, op. cit., pp. 44-46; l'auteur cite Pierre Coste, Saint Vincent de Pau. Correspondance, entretiens, documents, Paris, Gabalda, 1920-1925, 14 volumes ( $c f$. XI, 4 ; XII, 8 ; XII, 86). 
Louise de Marillac. Ses biographes disent que Monsieur Vincent savait parler aux hommes ${ }^{61}$ et que jamais homme n'a été écouté, suivi, obéi comme lui. Ses conférences du mardi ont un grand succès. Sous son autorité "d'éducateur de la charité » ${ }^{62}$, des "grands » s'engagent dans les bonnes œuvres et l'aident à fonder ses missions, des dames embrassent la vie religieuse en entrant chez les Sœurs de la charité. On trouve aussi chez lui un rapport charismatique à l'argent. Par exemple, il remet à l'hôpital de la Charité une somme de quinze mille livres que le maître des Monnaies lui avait données pour son propre confort. L'économie de ses fondations obéit au même principe, les dons reçus doivent être totalement distribués aux pauvres. Pour trouver des subsides, il fonde de vastes groupements artisanaux ou agricoles qui sont des modèles pour les ouvriers et les paysans ${ }^{63}$. Il se défait de cures qui rapportent. Le carrosse qui lui est offert parce qu'il a des difficultés à marcher devient une voiture pour apporter des vivres aux pauvres. Il écrit : "Plaise à notre Seigneur vous faire trouver le dégoût des choses de ce monde par la grande connaissance que vous acquerrez de la vanité d'icelles ». Ce qui ne signifie pas un total désintérêt pour l'économie. Selon Michel Riquet ${ }^{64}$, « il n'ignore ni les variations du pouvoir d'achat de la monnaie, ni les fluctuations des changes (...). Il s'entend parfaitement à en faire bénéficier les œuvres d'assistance qu'il ne cesse d'organiser au fur et à mesure de ses besoins. Nul mieux que lui ne sait prévoir et réaliser l'équilibre d'un budget, non pour la satisfaction d'un esprit comptable mais parce que le bien-être et la vie des pauvres en dépendent ». Rien en cela d'incompatible avec le charisme car ce dernier peut s'accompagner d'une rationalité économique ${ }^{65}$. La confirmation du charisme par un groupe de tiers est évidente chez saint Vincent de Paul. Il a été suivi par une armée d'hommes et de femmes qui se sont mis au service des pauvres dans le monde (neuf cents missions à sa mort). Toutefois, son charisme trouve ses limites : on ne lui connaît pas d'autres miracles que les conversions nombreuses ou les retours massifs à la pratique religieuse. D'autre part, on ne lui connaît pas de conflits avec l'Église à laquelle il s'est toujours soumis.

On peut se demander si sa mission de charité est l'exercice d'un charisme de fonction. Or, nous l'avons dit plus haut, sa bonté motive un élan collectif qui dépasse de loin la considération pour les pauvres et les malades. Elle dépasse les «bonnes œuvres » qu'il peut susciter au nom de l'Église et des vertus chrétiennes telles qu'un prêtre peut le faire chez ses paroissiens. De ce point de vue, saint Vincent de Paul pose le même problème que Louis Grignion de Montfort ${ }^{66}$.

61. Robert Sabatier, Saint Vincent de Paul, op. cit., p. 51.

62. Selon l'expression de R. Sabatier, op. cit., p. 37.

63. Idem, p. 47.

64. Michel RiQuet, op. cit., p. 123.

65. Bien qu'en principe l'économie charismatique soit irrationnelle. Nous avons traité de l'économie du charisme dans "Naissance d'un prophétisme en société industrielle. Rationalité de marché et économie du charisme. À propos de Charles Taze Russell ", Mélanges de Sciences religieuses, XXXVI-3, Lille, Facultés catholiques, 1979, pp. 175-190.

66. Jean SÉGuY, Social Compass, op. cit., pp. 5-24. 
Ni charisme prophétique, ni charisme de fonction, nous penchons vers le charisme personnel privé. Celui-ci a été défini par Jean Séguy comme une qualité exceptionnelle, hors du commun, qui se situe avant toute intervention institutionnelle, hors de celle-ci ou au-delà de celle-ci. Il ne propose ni légitimité, ni obligations nouvelles à proprement parler, la nouveauté qu'il propose reste toujours relative et interne au cadre institutionnel, c'est-à-dire acceptable par lui : il « renouvelle et interprète les obligations et la légitimité de l'institution à laquelle son porteur se rattache ${ }^{67}$. En somme, il réinterprète plus qu'il n'invente. Ce type de charisme remplit des fonctions renvoyant à la vie privée ; il se révèle capable d'effets collectifs de manière secondaire seulement ${ }^{68}$. Les fondateurs d'ordres religieux subordonnés à l'Église relèvent de ce type.

Le charisme personnel privé et le charisme de fonction peuvent se retrouver dans le même personnage ${ }^{69}$, un charisme personnel latent pouvant même être développé de façon à permettre à un porteur de charisme de fonction de légitimer des effets sociaux ne relevant pas de sa fonction ${ }^{70}$.

Le charisme de la bonté devient une vocation qui se moule dans une éthique des bonnes œuvres chez les laïcs, "c'est-à-dire ceux sur lesquels prophètes et prêtres cherchent à influer sur le plan éthique " ${ }^{71}$ et dans une gestion rationnelle de la charité comportant des fondations religieuses, des missions, des règlements, une économie des revenus et des prestations sociales.

\section{Des charismes spécifiques}

À la fin de cette mise en perspective et de l'illustration du charisme de la bonté avec la figure de saint Vincent de Paul, une première remarque s'impose : les charismes spécifiques présentent un double aspect. Comme tout charisme, ils n'existent que parce que des acteurs sociaux les reconnaissent mais Weber laisse l'impression qu'ils ont une existence subjective et sont manipulables. Ceci se manifeste particulièrement dans les domaines de l'esthétique de l'artisanat, de l'illumination, de la foi et de la bonté. Les charismes qui s'y rattachent peuvent être éveillés et perfectionnés par la volonté du croyant (comme le charisme de la foi) ou par l'ascèse qui - nous l'avons dit plus haut - comporte la mortification de l'orgueil, la libération des impressions du monde sensible et des abstractions de l'entendement, la formation d'un habitus social de la personnalité. À lire Weber, les charismes spécifiques relèveraient à la fois d'une économie sociale et

67. Jean SÉGUY, «Le clergé dans une perspective sociologique ou que faisons nous de nos classiques ", op. cit., p. 40.

68. Jean SÉGuY, Social Compass, op. cit., p. 9.

69. Idem, p. 6.

70. Idem, p. 8 .

71. Max Weber, Économie et société, op. cit., p. 463 ; Voir aussi M. Weber, Essais sur la théorie de la science, Paris, Plon (trad. J. Freund), 1965, p. 330-331. 
d'une économie personnelle. Cette dualité renvoie à la discussion sur le rôle de la dimension personnelle dans la formation des activités sociales dont l'auteur traite dans sa sociologie compréhensive ${ }^{72}$. D'autres aspects contribuent à leur définition : leurs rapports à la domination, à la magie et à la virtuosité.

\section{Charisme prophétique, charisme de fonction et charismes spécifiques sous l'angle de la magie}

Weber discute de la différence entre charisme de fonction et charisme prophétique mais comme il ne définit pas les charismes spécifiques, il ne discute pas la différence entre ceux-ci et les deux premiers. Peut-être parce que les charismes spécifiques n'ont pas la puissance transformatrice des premiers ? Mais rien n'empêche de mettre ces trois types de charisme en perspective, notamment à partir de deux critères, la magie et la domination.

Nous l'avons dit plus haut, Weber évoque un don extraordinaire et dans le paragraphe consacré au charisme prophétique ${ }^{73}$, il lui donne un contenu maximaliste. En effet, le prophète est un magicien beaucoup plus grand et plus puissant que les autres en ce qu'il possède non seulement un " pouvoir inouï sur les démons, mais aussi sur la mort elle-même ». En somme, il peut accomplir des choses que les autres magiciens sont incapables de faire. Les charismes spécifiques ne produisent pas ces effets extrêmes. Il existe dans le charisme prophétique wébérien une magie extrêmement puissante que l'on retrouve avec une intensité moindre dans sa forme routinisée, ce qui oblige l'auteur à comparer le prêtre, dont le charisme de fonction comporte également une qualification magique, au magicien ${ }^{74}$. Jean Séguy constate que Weber «a du mal à différencier le prêtre du magicien " ${ }^{75}$ mais il ajoute que "les trois spécialistes religieux prétendent bien à la possession et à la manipulation exclusive du charisme ». A priori ce n'est pas la magie qui distingue le prêtre du magicien mais les conditions de son exercice. Le prêtre est le « fonctionnaire d'une entreprise permanente régulièrement organisée en vue d'influencer les dieux, par opposition aux interventions personnelles et occasionnelles des sorciers ${ }^{76}$. Le charisme de fonction, typique du clergé, est légitimé par le charisme personnel du prophète mais il est dépersonnalisé et ne se manifeste pas par des dons surhumains pour légitimer une croyance ou des nouveaux commandements. Il en est un reliquat reçu et, s'il permet de contraindre les dieux par des sacrifices et par la prière, il n'a pas la puissance d'un charisme prophétique ou uniquement magique, car son porteur se placerait hors d'une

72. Idem.

73. Max WEBER, Économie et société, op. cit., pp. 464-466.

74. Idem, p. 450.

75. Jean SÉGUY, "Le clergé dans une perspective sociologique ou Que faisons-nous de nos classiques ? ", op. cit., p. 24.

76. Max Weber, Économie et société, op. cit., p. 450. 
institution qui ne réclame de lui aucune innovation. La qualification magique des charismes spécifiques est plus discutable. La magie est mentionnée par Weber dans deux cas, celui du charisme de la foi qui procure un «succédané des facultés magiques ${ }^{77}$ et dans celui du savoir-faire qui est magique à l'origine ${ }^{78}$. Ici, la formule doit être reliée à ses considérations sur «l'art » des artisans, d'abord empêtrés dans "les filets de la magie " dont le métier apparaissait comme un charisme personnel ou héréditaire et qui se sont ensuite défaits de leur caractère magique quand, installés dans les villes, ils ont dû penser rationnellement leur métier $^{79}$. On pourrait ajouter le cas du charisme de la vertu : celui-ci comporte une magie mais elle se limite à être apotropaïque. Les autres charismes spécifiques mentionnés plus haut ne semblent pas liés à la manipulation de forces supraempiriques. En effet, on ne voit pas en quoi les charismes de la bonté, de la chasteté, de la certitude du salut, de la raison, de l'illumination, de l'état de grâce et de l'esprit et du goût peuvent être magiques. Ils ne contraignent en rien les forces surnaturelles pour obtenir des résultats empiriques ; ils légitiment l'activité de leurs porteurs et éventuellement celle de leurs suiveurs, de leurs admirateurs ou de leurs bénéficiaires. Entre le charisme prophétique, le charisme de fonction et les charismes spécifiques, on trouve différents degrés de magie, de la plus puissante à la plus discrète.

\section{Les charismes spécifiques et la domination}

Nous constatons le même dégradé lorsque nous considérons les charismes sous l'angle de la domination. Le charisme est lié à la domination (certains interprètes de Weber, en particulier des politologues, semblent ne voir qu'un lien quasi exclusif entre les deux) et au changement ${ }^{80}$. Le prophète a un pouvoir usurpé, exercé directement sur ses disciples pour peu qu'ils confirment son charisme. Il délivre de nouveaux commandements et impose un nouveau style de vie à l'intérieur des limites de son aire d'influence. Le charisme de fonction fonde aussi un pouvoir. Séguy en traite ${ }^{81}$ : en reprenant Weber, il constate que le pouvoir des clercs, comme celui des prêtres et des prophètes, trouve sa source dans la possession et dans la manipulation exclusive du charisme. Celui que revendique le clerc est un charisme de fonction : impersonnel, transmis, il se légitime à l'intérieur de la tradition, à son profit et se reproduit. Le pouvoir du clergé vient de sa prétention à monopoliser la définition de ce qu'il faut croire et faire. On pense aux rites dont il croit détenir les instruments de leur efficacité. D'autre part, il en vient

77. Idem, p. 576.

78. Max Weber, Sociologie des religions, op. cit., p. 435.

79. Idem, p. 435.

80. Jean-Martin Ouedraogo, "Le charisme selon Max Weber : la question sociologique ", Archives européennes de sociologie, XXXVIII/2, 1997, pp. 329-334.

81. Jean SÉGUY, «Le clergé dans une perspective sociologique ou que faisons nous de nos classiques? ", op. cit. 
parfois à réguler la vie profane. Comme clerc, son pouvoir est limité par la force de l'institution qui lui procure un pouvoir. Ce dernier est plus faible que celui du prophète, il n'innove pas, ne produit pas les phénomènes extraordinaires cités plus haut. Il n'en est que sa routinisation ${ }^{82}$. Les charismes spécifiques semblent, quant à eux, généralement distants du phénomène de la domination. Hormis le charisme de la raison qui, chez Robespierre, légitime une prétention à dominer et celui de la vertu qui authentifie le monarque de la Chine ancienne en tant que « fils du ciel » mais qui, toutefois, ne conditionne pas l'accès à sa fonction, les autres charismes ne fondent pas un type de domination légitime.

On rencontre un écart entre la domination et les charismes spécifiques dans les mouvements pentecôtistes et charismatiques ainsi que dans les Églises indépendantes africaines (AIC). Chez les premiers, il existe une double hiérarchie : l'une, ministérielle (pasteurs, prédicateurs), l'autre spirituelle, fondée sur l'accumulation des dons chez les fidèles. Dans les secondes comme la Zionist Church, des healer prophets (le mot prophète n'est pas à prendre au sens wébérien) exercent un ministère de guérison. Ce sont souvent des guérisseurs traditionnels convertis au christianisme qui peuvent reprendre leur première fonction après une déconversion - seul l'appel de l'Esprit saint ou des ancêtres en décide. Selon Gerhardus C. Oosthuizen, leur ministère est considéré comme vital pour le bienêtre de l'Église dans le contexte africain ${ }^{83}$. Des fidèles des «Églises historiques » les consultent, augmentant de ce fait le prestige de leur congrégation ${ }^{84}$. Toutefois, peu de healer-prophets deviennent chefs de leur mouvement ${ }^{85}$. Pour plusieurs motifs : d'abord, leurs échecs pourraient les discréditer et leur Église perdre son crédit avec eux. S'ils avaient une position dominante, la communauté pourrait se scinder en suivant un healer-prophet plus doué. Ensuite parce que ces guérisseurs pourraient être des déviants doctrinaux : en recherchant des résultats empiriques, ils peuvent faire appel aux Esprits ancestraux et non au seul Esprit saint et donc exercer leur art en désaccord avec les Écritures. Le chef d'une congrégation doit savoir réguler l'orthodoxie doctrinale et régler les problèmes que la congrégation rencontre avec un guérisseur religieux. Enfin, beaucoup de ces healer-prophets sont des femmes qui, bien que très actives, seraient écartées du pouvoir ${ }^{86}$ selon l'habitus culturel, même si la constatation est quelque peu nuancée par M. Mkhise ${ }^{87}$.

82. Max Weber, Économie et société, op. cit., pp. 253-255.

83. Gerhardus C. Oosthuizen, The Healer-Prophet in Afro-Christian Churches, Leiden, E.J. Brill, 1992, p. IX.

84. Idem, pp. 70-71.

85. Idem, p. 178.

86. R.H. FrIESEN, "Origin of the spiritual healing churches in Botswana ", in C.G. Oosthuizen, M.C. Kritshof, S.W. Dube, eds., Afro-Christianity at The Grassroots, Leiden, E.J. Brill, 1994, pp. 37-50, p. 46; R.J. CAzZIoL, "The role of women in the leadership of Zwaziland ", idem, pp. 211-227, p. 224.

87. M. MKHISE, "Toward the emancipation of women in a post-apartheid South Africa: a case of a prayer woman in a Zionist Church ", in Afro-christianity at The Grassroots, op. cit., pp. 195-204. 
Mais les religio-thérapeutes masculins des AIC le sont aussi. Globalement, le ministère de guérison reste subordonné à une autorité supérieure. Quand le guérisseur spirituel perd son don ou quand il abandonne sa croyance, un autre le remplace, l'essentiel étant, dans l'optique de ces Églises, que l'Esprit saint continue à souffler sur le mouvement.

Toutefois, cette dissociation entre charisme spécifique et domination ne se retrouve pas totalement dans les Églises de guérison. Ainsi, le comité directeur de la Science chrétienne est composé de praticiens même si ses statuts ne l'imposent pas. L'antoinisme est dirigé par le collège des desservants, lesquels sont tous des guérisseurs. La double hiérarchie temporelle et spirituelle est ainsi évitée. La raison en est que, d'une part, ces mouvements placent au centre de leur préoccupation le traitement spirituel des maladies, les guérisseurs en sont donc le pilier central et nécessaire. D'autre part, la possession d'un charisme de guérison éveillé irait de pair avec l'acquisition d'un haut degré de spiritualité permettant d'avoir le discernement et la capacité nécessaires à toutes fins pratiques ${ }^{88}$.

Les observations qui précèdent étonneront sans doute. Nous l'avons dit plus haut, les chercheurs, en particulier les politologues, nous ont habitués à envisager le charisme dans son rapport à la domination. Weber lui-même l'a fait en parlant de la domination charismatique bien qu'il en donne les limites. Or, les charismes spécifiques illustrent des charismes pas nécessairement liés à la domination. Ils peuvent être ainsi référés à l'excellence dans l'exercice d'une vertu morale ou dans l'utilisation et l'illustration d'une tradition religieuse ou laïque. Ils représentent quelque chose comme de l'extra-quotidien. Leur cas nous contraint à situer les charismes sur une échelle virtuelle au bout de laquelle il n'est plus question de domination.

\section{Les charismes spécifiques et la virtuosité}

Le charisme plénier du prophète fait de celui-ci un virtuose absolu. Les charismes spécifiques ne concernent qu'un domaine particulier (la bonté, la foi, le savoir-faire...) où leurs porteurs deviennent des virtuoses. Lorsqu'ils sont religieux, ces derniers sont des « instruments de Dieu », dépouillés (ou presque) de tous les moyens de salut magiques, qui cherchent leur salut par la qualité éthique d'une action voulue par Dieu dans le monde ${ }^{89}$. Leur virtuosité, à l'origine magique également, "soumet les pulsions naturelles à une conduite systématique de la vie, mais encore elle soumet à une critique radicale, éthico-religieuse, ses relations avec la communauté sociale et des vertus, qui sont inévitablement utilitaires et

88. Régis Dericquebourg, "Construction d'un type idéal des religions de guérison à partir d'un échantillon de groupes religieux minoritaires ", in Raymond MAssé et Jean BENOIST, éd., Convocations thérapeutiques du sacré, Paris, Karthala, 2002, chap. 2, pp. 39-59.

89. Max Weber, Sociologie des religions, op. cit., p. 363. 
conventionnelles et nullement hérö̈que ${ }^{90}$. Cette virtuosité peut être vécue comme un " devoir moral » imposé pour transformer le monde selon ses idéaux ascétiques. Elle devient alors celle du réformateur ou du révolutionnaire. Weber parle de l'éthique du virtuose qui comme le charisme magique (ici il fait un parallèle) "réclame constamment la confirmation de la virtuosité " ${ }^{91}$. L'auteur évoque dans le même paragraphe quelques types de virtuose : le virtuose de l'ascétisme, du martyre passif, de la vertu religieuse exercée en ce monde à l'intérieur d'une profession, de la légalité formelle, de la bonté acosmique comme chez saint François d'Assise ${ }^{92}$. Dans son énumération, nous reconnaissons des virtuosités liées à des charismes mentionnés plus haut tels que « la chasteté comme charisme personnel du virtuose religieux $"{ }^{93}$ (virtuose ascétique), le charisme de certitude du salut (virtuose de la vertu religieuse exercée dans le monde par la profession). Ailleurs, on lit que le charisme de la foi produit un virtuose de la foi ${ }^{94}$. Le porteur de charisme spécifique n'est donc a priori pas un prophète, ni un clerc, ni un « homme moyen religieux ».

Mais les concepts wébériens ne s'appliquent pas uniquement à la religion. Les charismes laïques illustrent les virtuoses d'une cause ou d'un mode de vie posés comme un idéal. Il en est ainsi des virtuoses voulant révolutionner le monde selon la Raison, ou encore des virtuoses de la jouissance des biens de ce monde qui, dans une société imprégnée d'une éthique du refus du monde, peut être une provocation (parfois héroïque) ainsi que des virtuoses de l'art au service d'une tradition. Cette virtuosité est aussi une habileté comme l'est par exemple la « virtuosité dans la torture de soi-même " que Weber évoque à propos d'un certain ascétisme monacal $^{95}$. Dans le domaine religieux, Weber compare la virtuosité à l'« oreille musicale» pour la religion. Au plan laïque, on pourrait dire qu'elle caractérise « celui qui s'y entend » pour construire une forme de vie exemplaire, fondée sur une éthique philosophique ou politique dans laquelle il se réalise pour élaborer son salut mondain de la même façon que le virtuose religieux élabore méthodiquement son salut. On trouve là une analogie qui ferait de ces charismes spécifiques laïques des succédanés des charismes spécifiques religieux.

À ce stade, nous conclurons que le charisme plénier qualifié d'authentique est celui des prophètes et des héros qui inventent des voies de salut ; les charismes spécifiques sont ceux des virtuoses qui excellent dans l'un des aspects du nouvel ordre du monde proposé par les premiers.

90. Max Weber, Économie et société, op. cit., p. 555.

91. Idem, p. 554.

92. Idem, p. 554.

93. Idem, p. 608.

94. Idem, p. 576.

95. Max WeBer, L'éthique protestante et l'esprit du capitalisme, op. cit., p. 147. 


\section{Discussion - conclusion}

Chez Weber, les charismes spécifiques spécifient une grâce appliquée avec une virtuosité à un domaine de la vie religieuse ou profane. Ils expriment une vertu morale, une disposition naturelle ou une technique acquise pratiquée avec un succès reconnu ou encore une virtuosité acquise (et dont leur porteurs deviennent les figures emblématiques) dans l'utilisation d'une tradition et tendent à innover dans ce cadre en proposant par exemple des nouvelles techniques d'ascèse, de nouvelles techniques artistiques, des organisations charitables. Ils ne situent pas leurs porteurs dans l'extra-quotidienneté. Ils seraient plutôt une grâce qui fonde une virtuosité dans le domaine du quotidien.

Ils sont une "affaire personnelle " parfois soumise à une institution comme le charisme de la bonté chez saint Vincent de Paul. Ils peuvent réinterpréter la fonction du clerc comme le charisme de la foi. Ils peuvent être une transfiguration du charisme religieux comme le charisme de la Raison invoqué par Robespierre. Ils paraissent une dérive du charisme prophétique et du charisme de fonction que l'on peut utiliser avec profit. Les sociologues peuvent en construire de nouveaux pour spécifier de nouvelles conduites sociales mais aussi pour qualifier les figures emblématiques du passé et du présent. Nous pensons, par exemple, à un charisme de la conversion ${ }^{96}$ pour spécifier une personnalité dotée d'une capacité peu ordinaire à conduire des personnes vers la foi. En effet, nous admettons que « la modernité et la rationalité en finalité qui l'accompagne ne parviennent pas à éliminer le charisme de l'histoire contemporaine » et que " le charisme peut toujours surgir, de manière imprévue, et s'affirmer comme s'affirme la rationalité en valeur de l'éthique de conviction $"{ }^{97}$. Ceci vaut pour les charismes spécifiques car l'exemple de la thérapie religieuse (pentecôtistes, charismatiques, scientistes chrétiens, Invitation à la vie, antoinistes) pour ne prendre que celui-là ${ }^{98}$, montre que des personnages détenteurs d'une grâce introduisent mezzo voce de l'extraordinaire dans le quotidien rationalisé.

Enfin, Weber lie indiscutablement les charismes à la domination et à la magie. Toutefois il semble qu'en inventant certains charismes spécifiques, il ne s'arrête pas là. Ces derniers semblent montrer qu'il existe une échelle des charismes au bout de laquelle il n'est plus question de la domination et peu question de la magie. Les charismes en général ne sont pas uniquement une affaire de pouvoir.

96. Comme le suggérait Philipe Barbey dans son mémoire de DEA, Les Témoins de Jéhovah. La survivance du christianisme antitrinitaire: une résistance spirituelle pour la foi en un Dieu unique, EPHE, $\mathrm{V}^{\mathrm{e}}$ section, 2001.

97. Jean SÉGUY, "Max Weber : agir, savoir et savoir pourquoi ", in Archives de Sciences Sociale des religions, 96, 1996, pp. 17-39. La citation (p. 25) résume les apports des publications incluses dans l'Annuaire 1993 publié par l'Association italo-allemande de l'université de Trente.

98. Nous pensons aussi au charisme de la parole chez les téléévangélistes. 
Le charisme est reconnu par des groupements humains pour autant qu'il puisse servir à leur donner du pouvoir, mais globalement, ils constituent peut-être des chances typiques (des occasions) que saisissent des hommes pour inventer, réveiller, réorienter des ordres et des styles de vie. En somme, les hommes reconnaîtraient les charismes et leur donneraient un destin en fonction du sens qu'ils souhaitent donner à l'action collective. Il reste au sociologue à expliquer, dans chaque cas, pourquoi «cela s'est fait ainsi et pas autrement ${ }^{99}$ ».

Régis DERICQUEBOURG

Groupe de Sociologie de la Religion et de la Laïcité. IRESCO-CNRS Université Charles De Gaulle, Lille 3

\section{Résumé}

Dans l'œuvre de Max Weber, nous trouvons la mention de "charismes spécifiques" qui n'ont apparemment pas suscité la curiosité des sociologues. Pour combler ce manque, nous avons tenté de la définir à partir des citations de Max Weber où ils apparaissent. Nous nous sommes attardé sur le charisme de la bonté en prenant l'exemple de Vincent de Paul, une figure emblématique du charisme de la bonté. Il apparaît que les charismes spécifiques "spécifient" une grâce appliquée avec une virtuosité à un domaine de la vie religieuse et profane. Ils expriment une vertu morale, une disposition naturelle ou une technique acquise dans l'utilisation d'une tradition à l'intérieur de laquelle ils tendent à innover. Ils ne situent pas leurs porteurs dans l'extra-quotidienneté mais sont une grâce qui fonde une virtuosité dans un domaine du quotidien où ils continent à émerger. Ils ne sont pas nécessairement magiques et ne légitiment pas nécessairement une domination. Ils invitent à réfléchir sur le charisme tel qu'il a été interprété jusqu'à présent. Le charisme spécifique dont Max Weber ne donne pas de type idéal est un outil dans les mains des sociologues qui veulent interpréter des conduites de virtuosité peu ordinaires dans la vie quotidienne.

Mots-clés : Charisme, charisme spécifique, virtuosité, domination, magie, Max Weber.

99. Pour reprendre la formule de Weber dans Gesammelte Aufsätze zur Wissenschaftlehre citée par Wilhelm Hennig, La problématique de Max Weber, Paris, PUF, 1996, p. 85-86. 


\section{Abstract}

In his writings, Max Weber mentions several charismas named "specific charisma". The sociologists seem to ignore them. So, trying to fill this gap, we give a definition based on quotations in which they appear. We have dedicated a long section to Vincent de Paul who illustrates the charisma of goodness. It seems that the specific charismas specify a grace applied to a part of the religious or the profane life. They emphasize a moral virtue, a personal disposition or a learned technique in the use of a tradition in which they tend to bring in some novelties. They do not inscribe their owners out of the daily life but they are a grace which gives virtuosity to the daily life in which they continue to appear. They are not necessary magic and they do not necessarily legitimise domination. They invite to revise the commonly accepted definition of the charisma. Max Weber does not construct an ideal type of the specific charisma which nevertheless can become a tool for the sociologists in order of interpreting non ordinary activities in the daily social life.

Key words: Charisma, specific charisma, virtuosity, domination, magic, Max Weber.

\section{Resumen}

En la obra de Max Weber encontramos la mención de "carismas específicos" que no han aparentemente suscitado la curiosidad de los sociólogos. Para subsanar tal ausencia, hemos intentado definirla a partir de las citas de Max Weber alli donde éstas aparecen. Nos detuvimos en el carisma de la bondad tomando el ejemplo de Vicente de Paul que es una figura emblemática del carisma de la bondad. Surge de alli que los carismas específicos "especifican" una gracia aplicada con un virtuosismo a un ámbito de la vida religiosa y profana. Expresan una virtud moral, una disposición natural o una técnica adquirida en la utilización de una tradición al interior de la cual tienden a innovar. No sitúan a sus portadores en la extra-cotidianidad, sino que son una gracia que funda un virtuosismo en un ámbito de lo cotidiano donde emergen. No son necesariamente mágicos, y no legitiman necesariamente una dominación. Invitan a reflexionar sobre el carisma tal como ha sido interpretado hasta el presente. El carisma específico del que Max Weber no da un tipo ideal es una herramienta en manos de los sociólogos que quieren interpretar conductas de virtuosismo poco ordinarias en la vida cotidiana.

Palabras clave : carisma, carisma especifico, virtuosismo, dominación. 
